\title{
CARACTERÍSTICAS DEFINIDORAS DE TRAUMA VASCULAR PERIFÉRICO EM URGÊNCIA E EMERGÊNCIA: OCORRÊNCIA E TIPOS
}

\author{
Defining characteristics of Peripheral vascular trauma in Urgent and Emergency: \\ occurrence and types \\ Definición de las características del trauma vascular periférico en urgencia y emergencia: \\ la aparición y los tipos
}

Paula Krempser $^{1}$

Cristina Arreguy-Sena ${ }^{2}$

Ana Paula Sobral Barbosa ${ }^{3}$

\section{RESUMO}

Pesquisa tipo convergente assistencial. Objetivou-se calcular a ocorrência e documentar as evidências clínicas de trauma vascular em punções periféricas de adultos e idosos em um serviço de urgência e emergência de Minas Gerais, Brasil. Foram considerados como critérios de inclusão: o primeiro demítrio puncionado e a realização de avaliações clínicas em intervalos inferiores a 24 horas. As evidências de trauma foram registradas por fotografias. Amostra de seleção completa. Dos 200 sítios avaliados, houve 110 casos de trauma vascular com 288 manifestações de: dor, alteração da coloração da pele, diminuição da capacidade funcional local, edema, enduração, solução de continuidade, pustulação e hipo ou hipertermia local. As fotografias documentaram cada tipo de manifestação passível de registro visual. Tal investigação possibilitou reafirmar características definidoras para o diagnóstico de enfermagem "Trauma vascular periférico" em um serviço de Urgência/Emergência.

Palavras-chave: Enfermagem. Vasos sanguíneos. Diagnóstico de enfermagem. Vulnerabilidade.

\begin{abstract}
Convergent assistance research. The objective was to estimate the occurrence and document the clinical evidence of peripheral vascular trauma in punctures of adults and elderly in an urgency and emergency department in Minas Gerais, Brazil. We considered the following inclusion criteria: the first side punctured and the clinical assessments at intervals of less than 24 hours. The evidences of trauma were recorded by photographs. Sample with a full selection. From the 200 sites evaluated, there were 110 cases of vascular trauma with 288 events of: 1) pain, skin discoloration, local functional capacity decreased, edema, induration, cuts, postulation and local hypo or hyperthermia. The photographs documented every type of event capable of visual record. This investigation led us to reaffirm the defining characteristics for nursing diagnosis of "pheripheral vascular trauma" in the Urgency/Emergency department.
\end{abstract}

Keywords:Nursing. Blood vessels. Nursing Diagnosis. Vulnerability.

\section{Resumen}

Investigación de tipo convergente asistencial. El objetivo fue calcular la aparición y documentar la evidencia clínica del trauma vascular periférico en las punciones de adultos y ancianos en el departamento de urgencia y emergencia de Minas Gerais, Brasil. Se consideraron los siguientes criterios de inclusión: el primer lado perforado y la evaluación clínica a intervalos de menos de 24 horas. Las evidencias de trauma fueron registrados en fotografías. Muestra con selección completa. De los 200 sitios estudiados, había 110 casos de trauma vascular con 288 de los acontecimientos de: dolor, decoloración de la piel, disminución de la capacidad funcional local, edema, endurecimiento, cortes, postulación e hipo o hipertermia local. Las fotografías documentan todo tipo de eventos capaces de registro visual. Esta investigación nos ha permitido reafirmar las características definitorias de los diagnósticos de enfermería de "traumatismo vascular periférico" en el servicio de Urgencias/ Emergencias.

Palabras clave: Enfermería. Los vasos sanguíneos. Diagnóstico de Enfermería. Vulnerabilidad.

\footnotetext{
${ }^{1}$ Enfermeira Graduada pela Faculdade de Enfermagem da Universidade Federal de Juiz de Fora e integrante do Grupo de Pesquisa "Tecnologia, Cultura e comunicação em Saúde e em Enfermagem". Juiz de Fora - MG. Brasil. E-mail: paula@krempser.com.br ; ${ }^{2}$ Enfermeira, Doutora e Professora Associada da Faculdade de Enfermagem da Universidade Federal de Juiz de Fora. Juiz de Fora - MG. Brasil. E-mail: cristina.arreguy@ufjf.edu.br; ${ }^{3}$ Estatística, Doutora e Professora Adjunta da Universidade Fluminense, Rio de Janeiro, Brasil. Saquarema - RJ. Brasil. E-mail: ana_sobral@vm.uff.br
} 


\section{INTRODUÇÃO}

As punções venosas periféricas, características da assistência clínica pós-moderna, representam, aproximadamente, $85 \%$ das atividades executadas pela equipe de enfermagem em $50 \%$ das pessoas internadas ${ }^{1-2}$. Elas possibilitam: administrar líquidos e eletrólitos intravenosos; realizar exames bioquímicos e diagnósticos; infundir princípios farmacológicos; ofertar suporte nutricional parenteral, realizar hemotransfusão, monitorar hemodinâmica e viabilizar terapia renal substitutiva ${ }^{1,3}$.

Envolvem alto nível de complexidade técnico-científico, justificado por se tratar de um procedimento invasivo, romper a proteção natural da pele e comunicar o sistema vascular com o meio externo ${ }^{3-4}$. Tal fato requer a conciliação de competência profissional, conhecimento especializado, habilidade psicomotora, atualização com inovações tecnológicas e tomada de decisão $0^{3-4}$.

Apesar do benefício, uma pessoa que tem seu vaso puncionado pode apresentar trauma vascular. Ele está sendo concebido como o estado no qual o indivíduo apresenta "danos internos ou externos (lesando diretamente a estrutura do vaso ou desencadeando impacto sobre áreas próximas a ele, quer seja de origem química e/ou bioquímica e/ou física e/ou biológica), em uma ou várias camadas teciduais, ou em tecidos subjacentes"3.

Entre as unidades assistenciais em que os vasos são manuseados estão os Serviços de Urgência/Emergência, ou seja, unidades destinadas ao atendimento de pessoas com agravamentos agudizados que requerem a intervenção imediata de equipe multiprofissional especializada. 0 Pronto-Socorro, um tipo de unidade de emergência destinada à prestação de assistência a pessoas doentes, com ou sem risco de vida, por definição, requer dos profissionais que atuam nestes cenários que eles coletem dados para caracterizar as situações que coloquem a vida dos usuários em risco, que tomem decisões rápidas e que adotem intervenções precisas ${ }^{5-6}$.

As evidências que permitem considerar o trauma vascular na perspectiva de um diagnóstico de enfermagem são subsidiadas pelas seguintes argumentações: o parecer do Enfermeiro sobre o tipo, a qualidade e a adequação dos materiais aos indivíduos que terão suas veias puncionadas; a escolha do local, do tipo de cateter e da forma de fixá-lo; a identificação de evidências que subsidia a tomada de decisão de enfermeiros para repunção de vasos sanguíneos quando apresentam evidências de trauma vascular, dentre outros ${ }^{1 ; 3 ; 7-}$ ${ }^{10}$, não havendo evidências suficientes sobre a temática para afirmá-la como um diagnóstico de enfermagem na realidade dos Serviços de Urgências e Emergências.

Investigar a ocorrência de trauma vascular periférico no contexto do atendimento de urgência e emergência adquire um novo dimensionamento que permite considerá-la como o objeto da presente investigação, tendo em vista a existência de lacuna deste enfoque na literatura.

Diante do exposto, a presente investigação objetivou calcular a ocorrência e documentar as evidências clínicas de trauma vascular em punções periféricas de adultos e idosos em um serviço de urgência e emergência. A justificativa alicerçouse na necessidade de: 1) delinear as peculiaridades do processo de punção nesta realidade; 2) validar clinicamente características definidoras compatíveis com a existência do diagnóstico "Trauma vascular periférico", 3) subsidiar a redefinição de situações passíveis de ser abordadas com condutas terapêuticas de enfermagem e 4) gerar novos conhecimentos capazes de qualificar a assistência de enfermagem.

\section{MÉTODOS}

Pesquisa tipo convergente assistencial ${ }^{11}$. A amostra de seleção completa foi composta por sítio de punções vasculares em pessoas atendidas em um Serviço de Urgência e Emergência, e a finalidade das punções realizadas pela primeira vez em cada estrutura corporal (demítrio direito ou esquerdo) do participante foi o tratamento farmacológico ou coleta de sangue para realização de exames laboratoriais. Pesquisa composta por 200 punções vasculares periféricas ocorridas durante dois meses (março e abril/2011). Os participantes foram recrutados ao adentrarem na Unidade de Pronto-Socorro no período das $18 \mathrm{~h}$ às $21 \mathrm{~h}$. Foram critérios de elegibilidade: pessoas de ambos os gêneros, maiores de 18 anos de idade, todas as raças e cores de pele, que tiveram seus vasos puncionados (no atendimento do SAMU ou na Instituição) em situação de urgência ou emergência. 0 critério de início da participação ocorreu a partir da punção de um vaso. 0 critério para interrupção do acompanhamento na investigação ocorreu na remoção do cateter, por ocasião da alta ou após remissão completa das evidências de trauma vascular, quando instaladas.

Cada sujeito pôde entrar por até duas vezes na investigação, quando as punções foram realizadas em demítrios distintos. Foram excluídas as pessoas que se recusaram ou que não apresentavam condições para assinar o Termo de Consentimento livre e Esclarecido pós-informado e aquelas que tiveram os vasos puncionados em um mesma estrutura corporal por segundo ou mais tentativas. Foram utilizados os critérios e os instrumentos de coleta de dados propostos por ArreguySena (2002) para captar a ocorrência de trauma vascular, a saber: 1) caracterização dos participantes; 2) identificação e mensuração das variáveis intervenientes; 3 ) identificação da variável de desfecho (trauma vascular) e 4) registro fotográfico do trauma em curso.

A utilização de um instrumento previamente testado e validado favoreceu a captação dos tipos de traumas vasculares, e a presença de definições operacionais previamente validadas permitiu delinear o evento avaliado com segurança. 
Foram realizadas avaliações clínicas diárias (técnicas semiológicas e registros fotográficos) com intervalos inferiores a 24 horas entre duas avaliações subsequentes. 0 número de avaliações realizadas em cada sítio variou com o tempo de permanência do cateter, com a duração e remissão da manifestação de trauma vascular periférico, e foi influenciado pela alta hospitalar. Isso equivale a dizer que a média de dias de avaliação do sítio foi de 2,39 dias (com variabilidade de menos de um dia a 34 dias).

Os dados foram consolidados em planilha no Statistical Package for the Social Sciences (SPSS), tratados com frequência simples e percentual e analisados à luz de referenciais teóricos que abordam a temática.

0 projeto foi submetido ao Comitê de Ética da Universidade Federal de Juiz de Fora (Parecer 295/2010), com atendimento de todos os aspectos éticos e legais de pesquisa envolvendo seres humanos.

\section{RESULTADOS}

Foram 200 sítios de punção de cateteres intravasculares periféricos avaliados em 179 pessoas adultas em um Serviço de Pronto-Socorro para fins terapêuticos e diagnósticos em primeira tentativa por demítrio.

Estas punções foram realizadas em: pessoas do sexo masculino (55\%); com idade média de 47 anos e mediana de 46 anos (variabilidade dos 20 aos 96 anos de idade); média de 2,39 dias de internação (variabilidade inferior a 24 horas e até 34 dias). A saída da investigação deveu-se: à alta da instituição, em 24 (12\%) clientes; à cura do fator que gerou a internação, em 91 (45,5\%); à melhora das condições de saúde, em 62 (31\%); à transferência de instituição hospitalar, em 21 (10,5\%); ou pedido ou fuga hospitalar, em dois (1\%).

0 processo de punção venosa periférico nos participantes foi realizado nas seguintes locais: 102 (51\%) foram no braço direito e 109 (54,5\%) na face anterior. Foram observadas 151 (75,5\%) punç̃̃es no antebraço, 45 (22,5\%) na mão e quatro (2\%) no braço, sendo $115(57,5 \%)$ na região lateral e 85 (42,5\%) na região medial.

0 posicionamento em relação à raiz do membro puncionado ficou assim caracterizado: 85 (42,5\%) foram proximais, $72(36 \%)$ mediais e $43(21,5 \%)$ em regiões distais, e o local de punção ocorreu em articulação da mão, do antebraço ou do braço em 104 (52\%) casos.

0 número de adesivos para fixação do cateter intravascular variou de um a oito, sendo mais observado o uso de três a quatro adesivos, $37(18,5 \%)$ e $62(31 \%)$ respectivamente. Em 200 complexos de fixação analisados, $141(70,5)$ deles utilizaram esparadrapo comum como material de fixação.

A troca do complexo de fixação ocorreu em 12 casos $(3,6 \%)$, sendo que, em $35(24,3 \%)$ deles, a fixação se apresentava com alguma sujidade. Em 40 (28,5\%) complexos de fixação, os sítios estavam úmidos. As razões da presença da umidade ficaram assim distribuídas: $25(64,1 \%)$ casos em decorrência do contato com água de banho; quatro (10,25\%), pelo contato com material biológico, tendo como marcador a presença de sangue visível; cinco (12,8\%), devido ao extravasamento de medicações vascular; e cinco (12,8\%), devido à presença de suor.

Considerando a finalidade da punção venosa, 109 (54,5\%) delas foram realizadas para terapêutica; 41 (20,5\%) para diagnósticos; 38 (19\%) foram medicamentosas e $12(6 \%)$ equivaleram a punções sem êxito. A designação da finalidade "medicamentosa" baseou-se na realização de infusões intermitentes. Cabe destacar que foi unânime o uso de agulhas do tipo flexíveis sem extensores nas punções realizadas com fins terapêuticos ou medicamentosos e para fins diagnósticos, $30(76,9 \%)$ punções utilizaram seringas e agulhas e nove (23\%) utilizaram o sistema de vácuo para a coleta de sangue.

0 calibre das agulhas utilizadas foi: $20 \mathrm{G} \mathrm{em} 80$ casos (40\%); $22 \mathrm{G} \mathrm{em} 115(57,5 \%)$ casos e $24 \mathrm{G} \mathrm{em}$ cinco $(2,5 \%)$ casos.

0 registro de enfermagem a respeito da fixação do cateter intravascular não foi realizado em 186 (93\%) casos.

\section{Tipos de características definidoras de trauma vascular periférico e sua ocorrência}

Foram identificadas as seguintes características definidoras: 1) dor na inserção e no trajeto do cateter; 2) diminuição da capacidade funcional local e 3) alterações da integridade da pele dos tipos: modificação na coloração; na estrutura; na integridade/solução de continuidade; no conteúdo e na temperatura local.

Em um total de 200 punções venosas periféricas, houve ocorrências de trauma vascular em 110 (55\%) participantes. Considerando que cada indivíduo pode apresentar mais de um tipo de manifestação de trauma vascular, obtivemos um total de $288(144 \%)$ registros de características definidoras de traumas vasculares periféricos.

0 tempo médio de permanência de um mesmo cateter intravascular no interior do vaso foi de 1,6 dia com variabilidade de períodos inferiores a 24 horas a sete dias. 0 tempo para identificação de pelo menos uma manifestação de trauma vascular periférico ficou assim caracterizado: 91 punções $(45,5 \%)$ evoluíram antes que se completassem 24 horas da punção; em 96 punções (48\%), o trauma manifestou-se no primeiro dia da instalação do cateter; em cinco punções (2,5\%), no segundo dia da punção; em outras cinco punções $(2,5 \%)$, no terceiro dia; e, em uma punção, no quarto $(0,5 \%)$, no quinto $(0,5 \%)$ e no sexto dia $(0,5 \%)$, respectivamente. $A$ média foi de 0,68/dia para a identificação de pelo menos uma evidência de trauma vascular periférico. 
Em relação à ocorrência dos 110 traumas avaliados, em 72 (65,5\%) deles foram mantidas as condições iniciais até o momento da alta institucional; em 12 (10,9\%) casos, eles aumentam; em $13(11,8 \%)$ houve regressão parcial de sua evolução e, em outros $13(11,8 \%)$, ocorreu a cura antes do período de alta hospitalar.

Das 200 punções venosas periféricas acompanhadas, $97(48,5 \%)$ indivíduos tiveram alta hospitalar com presença de trauma vascular em curso e $103(51,5 \%)$ indivíduos tiveram alta hospitalar sem evidências de trauma vascular.

A ocorrência de traumas vasculares nas 200 punções avaliadas consta da Tabela 1.

Dentre os 200 sítios de punções observados no setor de urgência e emergência, foram observados 110 casos $(55 \%)$ de trauma vascular periférico distribuídos em 288 (144\%) manifestações de traumas vasculares. Do total de características definidoras de trauma vascular periférico em decorrência do uso de cateter vascular, foram identificadas: 1) dor $(38,9 \%) ; 2)$ diminuição da capacidade funcional do membro puncionado ou parte dele $(2,8 \%)$ e 3$)$ alterações na integridade da pele, manifestadas por modificação: na estrutura - edema (19,1\%), enduração $(6,9 \%)$; na solução de integridade/continuidade $(6,3 \%)$; na coloração - hiperemia, equimose, hematoma $(20,8)$; no conteúdo - secreção no sítio de inserção do cateter $(0,7 \%)$ e na temperatura local - aumento ou diminuição $(4,5 \%)$, que são apresentadas na Tabela 1. A exemplificação fotográfica dos tipos de traumas vasculares periféricos consta na Figura 1.

Figura 1. Registros fotográficos de traumas vasculares periféricos. Juiz Fora, Jul/2011. Hematoma

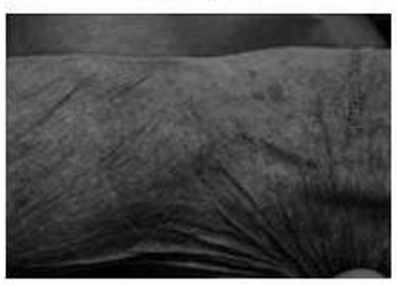

Eritema trajeto

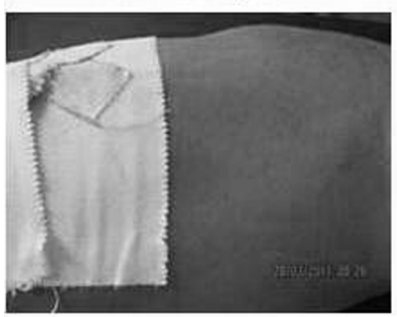

Cacifo

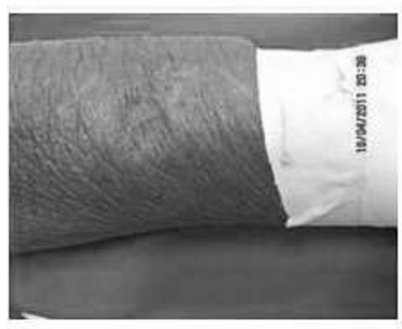

Endurado generalizado

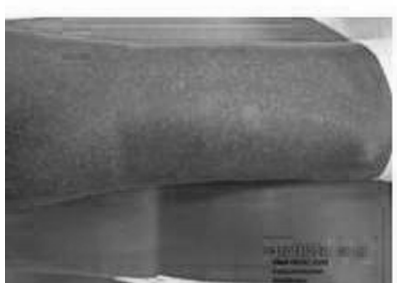

Equimose

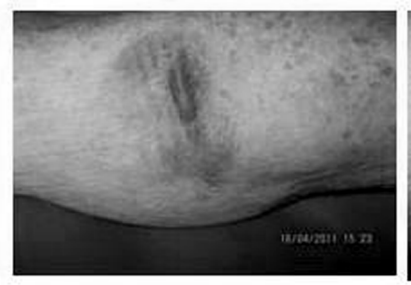

Edema local

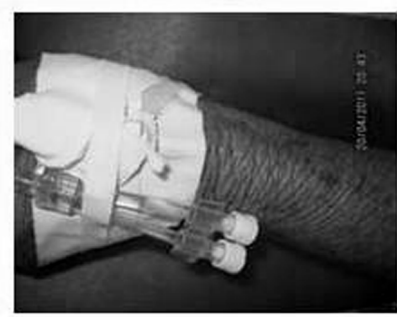

Endurado de trajeto

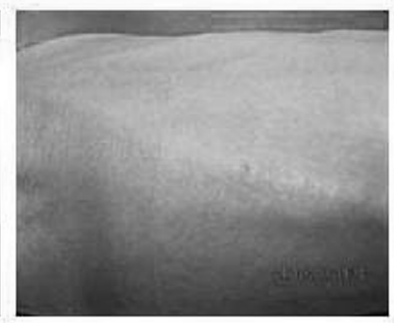

Solução continuidade

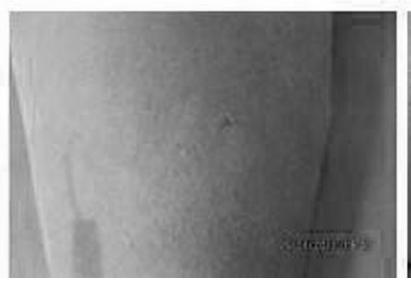

Eritema pontual

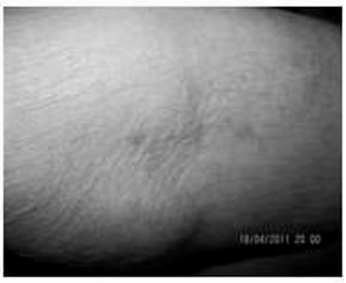

Edema generalizado
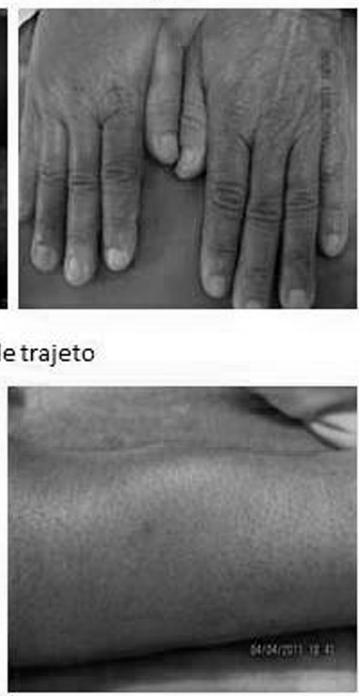

Secreção

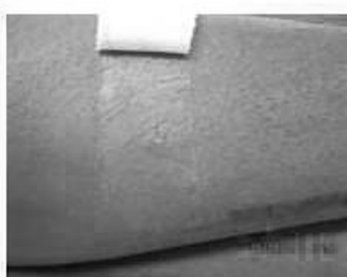


Tabela 1. Distribuição das 288 manifestações de traumas vasculares periféricos identificadas no processo de punção vascular, segundo tipos. Juiz de Fora, maio/2011.

\begin{tabular}{|c|c|c|c|c|c|c|}
\hline \multirow[t]{2}{*}{+2} & \multirow[t]{2}{*}{ TIPOS DE TRAUMA } & \multirow[t]{2}{*}{$\mathrm{n}$} & \multirow[t]{2}{*}{$\%$} & \multicolumn{2}{|c|}{ Variância medida } & \multirow[t]{2}{*}{ Média } \\
\hline & & & & Minimo & Máxima & \\
\hline \multirow[t]{2}{*}{ DOR } & Sitio inserção cateter & 76 & 26,3 & \multirow{2}{*}{1} & \multirow{2}{*}{7} & \multirow{2}{*}{1,4} \\
\hline & Trajeto venoso & 36 & 12,6 & & & \\
\hline \multicolumn{2}{|c|}{ CAPAQDADE FUNGONAL } & 8 & 2,8 & 3 & 5 & 4,9 \\
\hline \multicolumn{7}{|c|}{ ALTERAÇĀO INTEGRIDADE } \\
\hline \multirow{3}{*}{ 芯 } & Sitio inserção cateter & 40 & 13,9 & \multirow[t]{2}{*}{1} & \multirow{2}{*}{50} & \multirow{2}{*}{6,3} \\
\hline & Generalizado & 8 & 2,8 & & & \\
\hline & Cacifo & 7 & 2,4 & 1 & 4 & 2,7 \\
\hline \multirow{2}{*}{ 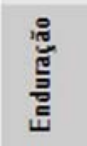 } & Generalizada & 2 & 0,7 & \multirow{2}{*}{2} & \multirow{2}{*}{16} & \multirow{2}{*}{4,6} \\
\hline & No trajeto venoso & 18 & 6,2 & & & \\
\hline \multicolumn{2}{|c|}{ Solução Continuidade pontual } & 18 & 6,3 & $20 G$ & $24 G$ & \\
\hline \multirow{5}{*}{ 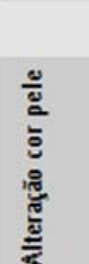 } & Pustulação & 2 & 0,7 & & & \\
\hline & Equimose & 29 & 10 & $1 \mathrm{~cm}^{2}$ & 25 & 4,9 \\
\hline & Hematoma & 4 & 1,3 & $14 \mathrm{~cm}^{2}$ & 105 & 51,2 \\
\hline & Enitema pontual & 16 & 5,6 & $20 G$ & $24 G$ & \\
\hline & Eritema de trajeto & 11 & 3,9 & $1 \mathrm{~cm}^{2}$ & 53 & 11,6 \\
\hline
\end{tabular}

Nota das autoras: A soma dos traumas perfaz valor superior a 100\% em decorrência de um mesmo sítio poder apresentar mais de uma manifestação.

\section{DISCUSSÕES}

Das pessoas que tiveram alta institucional pelos critérios médicos (melhora das condições de saúde; transferência de instituição; à pedido ou por fuga), 13 (6,5\%) delas alcançaram a cura do trauma instalado durante o período de acompanhamento da investigação, fato que remete à relevância de intervenções de enfermagem em tempo hábil para o controle da evolução e do agravamento do caso, mesmo em situações que envolvam um perfil institucional de pronto atendimento.

A análise do local de inserção dos cateteres intravasculares permitiu identificar o predomínio de punções realizadas no antebraço e na articulação. Considerando que a alteração do posicionamento articular se modifica no período de relaxamento, os locais de punções preferenciais utilizados constituem em sítio de risco para ocorrência de trauma vascular. Isso porque, na medida em que as estruturas corporais são flexionadas, elas intensificam a pressão intravascular, embora nem sempre este posicionamento culmine com a transfixação do vaso, em consequência da flexibilidade do material adotado

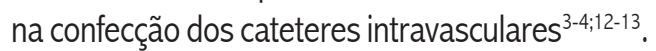

Há evidências de que substâncias cujos pHs são limítrofes para uso intravasculares ou são substâncias vesicantes são desaconselháveis para infusão nas veias das mãos ${ }^{3-4 ; 12-13}$. 0 posicionamento das punções intravasculares nas mãos de pessoas adultas e idosas é desaconselhável devido ao fato de os vasos serem tortuosos, não adesivos às estruturas adjacentes com fluxo minuto baixo quando comparados aos do antebraço $0^{13}$. As razões que alicerçam tais recomendações são: 1) 0 aumento da idade proporciona redução na quantidade de fibras elásticas, fazendo com que os envoltórios (camadas íntima e média dos vasos) engrossem e endureçam, gerando tortuosidades e intensificando as chances de uma transfixação por ocasião de sua punção e 2) a perda de fibras elásticas reduz a capacidade do vaso em suportar estresse, ficando suscetível à rotura e à dilatação (aneurisma), principalmente nas situações em que os vasos são submetidos a infusão de rápidos volumes de líquidos, a exemplo do que ocorre nos serviços de urgência/emergência ${ }^{4 ; 12}$. A escolha por posicionar as punções em antebraços vai de encontro às recomendações de literatura ${ }^{3-4 ; 13}$.

Há recomendações para que a seleção do local de punções atenda aos seguintes critérios: tipo de terapia prescrita, duração do tratamento, disponibilidade de sítios de acesso, diagnóstico médico, complicaç̃oes anteriormente identificadas no usuário, habilidade profissional do local de inserção, condições da pele, preferência e idade do usuário, dentre outros fatos que fazem com que as veias basílicas, cefálicas e braquiais sejam mais recomendadas ${ }^{3-4}$.

Numa classificação cuja finalidade foi analisar a seguranç̧a dos movimentos no processo de fixação de cateteres intravasculares periféricos de adultos, idosos e crianças, sugeriu-se: 1) a exclusão da prefixação (adesão do esparadrapo somente no cateter); 2) a substituição da fixação nula (adesão 
do esparadrapo somente na pele do usuário) pelo preparo da área de fixação; 3) a redução do número de fixações adicionais do tipo complementar (adesão do esparadrapo na pele e cateter simultaneamente); 4) a fixação do(s) extensor(es) para evitar trauma vascular pelo tracionamento do cateter ${ }^{14}$.

Há consenso sobre os benefícios do uso de coberturas transparentes estéreis devido à possibilidade de acompanhamento diário e redução da exposição a patógenos ${ }^{4}$.

0 fato de as faces anterior, lateral e proximal serem mais utilizadas como estrutura puncionada possibilitou que 0 complexo de fixação fosse atritado com a superfície do leito, intensificando as chances de seu deslocamento ${ }^{3}$, havendo respaldo para se evitar punções na face lateral em virtude da diminuição do risco de lesões de nervos 4 .

A presença de sujidade e de umidade foi considerada fator de risco para a ocorrência do diagnóstico de enfermagem "Risco para trauma vascular"15 e, quando associada ao rompimento da barreira natural da pele, ocasiona focos de infecção. A ocorrência de infecção na presente investigação foi influenciada pelo curto tempo de acompanhamento do cateter IV nos serviços de urgência/emergência - local de transição no contexto do cuidado hospitalar-4; ${ }^{3-3}$.

0 perfil dos cateteres intravasculares utilizados $(97,5 \%$ inferior ao 22G) foi ao encontro das recomendações da literatura para os materiais preconizados para uso em Serviços de Urgência/Emergência. Eles devem ser curtos e de grande calibre com vistas a compatibilizar o fluxo de reposição volêmica/minuto e estabilização hemodinâmica ${ }^{16}$. 0 fato de serem utilizados cateteres flexíveis nos sítios puncionados dificulta que os vasos sejam transfixados em casos de agitação psicomotora, mas intensifica a ocorrência de trauma por causa mecânica em decorrência do contato da agulha flexível com a íntima do vaso ${ }^{3 ; 13}$.

Considerando que o processo de fixação dos cateteres, quando realizado com esparadrapo comum ou hipoalergênico, oclui completamente o canhão do cateter (local de coloração que possibilita a identificação de seu calibre, segundo a regulamentação da Associação Brasileira de Normas TécnicasABNT $)^{17}$, impossibilitando a identificação do calibre do cateter, seu registro e a data de sua inserção são de fundamental importância no controle das punções vasculares realizadas em serviços que tenham grande rotatividade e demanda, como é 0 exemplo dos Serviços de Urgência e Emergência.

Há evidências de que a permanência de um cateter intravascular em um mesmo sítio não deva exceder 96 horas (quatro dias) desde que todo o período seja tutorado com avaliações periódicas para identificação precoce das evidências de trauma vascular-4; ${ }^{3-13}$

A ocorrência de trauma vascular com menos de 24 horas deve-se, em geral, à transfixação do vaso, quer seja instalado durante a punção, durante a mobilização da estrutura corporal ou em decorrência do deslocamento da agulha no interior do vaso ${ }^{3 ; 13}$.
0 registro completo de enfermagem incluiu os seguintes dados: nome do profissional que realizou a punção, a data, a hora e o calibre da agulha em sete $(3,5 \%)$ casos. Sua apreciação utilizou como parâmetro o conteúdo da Resolução 358/2009 que aborda sobre a sistematização da assistência de enfermagem tratando no artigo 6 sobre os registros profissionais como atividade obrigatória ${ }^{18}$

\section{CONCLUSÕES}

Ao analisar a ocorrência e documentar as evidências clínicas de trauma vascular em punções periféricas de adultos e idosos em um serviço de urgência e emergência, foi possível identificar $55 \%$ de ocorrência de traumas vasculares periféricos.

Foram documentadas três características definidoras para o trauma vascular periférico, a saber: 1) dor (38,9\%);2) alteração da capacidade funcional $(2,8 \%$ ) e 3 ) alteração da integridade por: modificação da estrutura da pele - edema e enduração (26\%), modificação na cor da pele $(20,8 \%)$; modificação na integridade/solução de continuidade $(6,3 \%)$; presença de secreção-pústula $(0,7 \%)$ e modificação da temperatura local $(4,5 \%)$.

Das três características definidoras categorizadas, 16 possibilidades de manifestações foram identificadas (evidências, sinais ou sintomas) e documentadas: 1) dor no sítio de inserção do cateter; 2) dor no trajeto venoso; 3 ) equimose; 4) hematoma; 5) eritema pontual; 6) eritema de trajeto; 7) alteração da capacidade funcional; 8) edema no sítio de inserção do cateter; 9) edema generalizado; 10) sinal de cacifo positivo; 11) endurado generalizado; 12) enduração no trajeto venoso; 13) solução de continuidade pontual; 14) pústula; 15) hipertermia local e 16) hipotermia local).

As lesões de trauma vascular periférico, compatíveis com registros fotográficos (hematoma, equimose, eritema pontual e de trajeto, edema local e generalizado, enduração de trajeto e generalizado, solução de continuidade e secreção) foram exemplificadas na presente investigação por fotografias e podem ser tratadas com cuidados de enfermagem não medicamentosos.

Do ponto de vista da prática de enfermeiros, a presente investigação permitiu documentar evidências de trauma vascular em curso, reafirmando a presença de características definidoras compatíveis com o diagnóstico de enfermagem "Trauma vascular periférico". 0 fato de $51,8 \%$ dos usuários terem alta institucional com pelo menos uma manifestação de trauma vascular em curso retrata uma realidade na qual 0 Enfermeiro possui respaldo (inter)nacional para prevenir a instalação do diagnóstico "Risco para trauma vascular" e a necessidade de que o cuidado de enfermagem seja contínuo, mesmo após a alta hospitalar, para os casos de "Trauma vascular periférico" a ponto de dar resolutividade para as manifestações de trauma instalados. 
A presente investigação, ao abordar o trauma vascular periférico em curso, na perspectiva de um diagnóstico de enfermagem, alerta os Enfermeiros para a necessidade de fazerem uma releitura deste procedimento à luz das novas tecnologias e conhecimentos. Os desafios apresentados pelo processo de punção de vasos para o Enfermeiro e sua equipe são: 1) a seleção dos sítios de maiores chances de viabilidade e de menores danos e 2) a identificação de manifestações de trauma vascular instaladas com vistas a direcionar o processo decisório para intervenções de enfermagem compatíveis com seu tratamento.

Diante do exposto, sugerimos que sejam realizados novos estudos de delineamento de coorte, caso controle e de prevalência, no sentido de identificar as associações e relações que possibilitam predizer sobre os fatores relacionados para a ocorrência de trauma vascular periférico em Serviços de Urgência/Emergência.

\section{REFERÊNCIAS}

1. Martins KA, Tipple AFV, Souza ACS, Barreto RASS, Siqueira KM, Barbosa JM. Adesão às medidas de prevenção e controle de infecção de acesso vascular periférico pelos profissionais da equipe de enfermagem. Ciênc. saúde coletiva.. 2008 out/dez; 7(4): 485-92.

2. Torres MM, Andrade D, Santos CB. Punção venosa periférica: avaliação de desempenho dos profissionais de enfermagem. Rev. Latino-Am. Enfermagem. 2005 maio/jun; 13(3): 299-304.

3. Arreguy-Sena C. Trajetória de Construção e validação do(s) diagnósticos: trauma vascular relacionado ao procedimento de punção venosa periférica e risco para trauma vascular relacionado ao procedimento a punção venosa periférica [tese doutorado]. Ribeirão Preto (SP): Escola de Enfermagem de Ribeirão Preto/USP; 2002.

4. Infusion Nurses Society. Infusion Nursing Standards of practice. J. infus. nurs.. 2011 jan/feb; 34(S1): 1458-533.

5. Montezelli JH, Peres AM, Bernaredino E. Demandas institucionais e demandas do cuidado no gerenciamento de enfermeiros em um pronto socorro. Rev. bras. enferm. 2011; 64(2): 348-354.

6. Kirchhof ALC, Magnago TS, Urbanetto JS, Cera MC, Marques CS, Capellari C. Os acidentes de trabalho atendidos em pronto- atendimento de hospital universitário. Esc Anna Nery. 2003 dez; 7(3): 361-8.

7. Arreguy-Sena C, Carvalho EC. Avaliação de punção venosa periférica: análise de critérios de remoção de dispositivo intravenoso adotados por uma equipe de enfermagem. Esc Anna Nery 2003 dez; 7(3): 351-60.

8. Fakih FT, Freitas GF, Secli SR. Medicação: aspectos ético-legais no âmbito da enfermagem. Rev. bras. enferm. 2009; 62(1): 132-5.
9. Santos JS, Scarpelini S, Brasileiro SLL, Ferraz CA, Dalloramelv, Sá MFS. Avaliação do modelo de organização da unidade de emergência do hcfmrpusp, adotando, como referência, as políticas nacionais de atenção às urgências e de humanização. Medicina ( Ribeirão Preto). 2003 abr/dez; 36: 498-515.

10. Wehbe, G; Galvão CM, 0 enfermeiro de unidade de emergência de hospital privado: algumas considerações. Rev. Latino-Am. Enfermagem 2001 mar/apr; 9(2): 86-90.

11 Paim L, Trentini M, Madureira VSF, Stamm M. Pesquisa convergenteassistencial e sua aplicação em cenários da Enfermagem. Cogitare enferm. $2008 \mathrm{jul} / \mathrm{set}$;13(3): 380-6.

12. Sociedade Brasileira de Cardiologia. Diretrizes da Sociedade Brasileira de Cardiologia: pocket book 2007-2010. Coordenação Jadelson Andrade. $3^{\circ}$ ed. rev. São Paulo(SP): OMNIFARMA; 2010.

13. Phillips LD. Manual of IV Therapeutics. 4th ed. Philadelphia (EUA): F.A. Davis Company; 2005.

14. Arreguy-Sena C, Carvalho EC. Trauma vascular de origem mecânica: categorização da fixação de cateteres endovenosos. Anais do 22 Congresso da Sociedade de Cardiologia do Estado de São Paulo - 19 Jornada de Enfermagem. 2002 mai. 2-4; Campos do Jordão(SP), Brasil. Campos do Jordão (SP): SOCESP; 2002. p.81.

15. Arreguy-Sena C, Carvalho EC. Risco de trauma vascular. In: NANDA International. Diagnósticos de enfermagem da NANDA: definições e classificação 2009-2011. Porto Alegre(RS): Artes Médicas; 2009.

16. Martin HS, Brandão Netto RA, Saclabrini Neto A, Velasco IT. Emergências Clínicas: abordagens práticas $4^{\circ}$ ed. Barueri(SP): Manole; 2009.

17. Associação Brasileira de Normas Técnicas - ABNT. NBR9753: Escalpe estéril e de uso único, requisitos e métodos de ensaio. Rio de Janeiro(RJ): ABNT; 1997.

18. Conselho Federal de Enfermagem (BR). Resolução COFEN n 358/ 2009: Dispõe sobre a Sistematização da Assistência de Enfermagem e a implementação do Processo de Enfermagem em ambientes, públicos ou privados, em que ocorre o cuidado profissional de enfermagem, e dá outras providências [citado 2012 Jun. 07]. Disponível em: <http:// site.portalcofen.gov.br/node/4384>. 